\title{
The Red Halos of Galaxies
}

\author{
Erik Zackrisson ${ }^{1,2}$, Nils Bergvall ${ }^{2}$, Göran Östlin ${ }^{3}$, Genoveva Micheva ${ }^{3}$, \\ and Brady Caldwell ${ }^{2}$ \\ ${ }^{1}$ Tuorla Observatory, Väisäläntie 20, FIN-21500 Piikkiö, Finland, email: ez@astro.uu.se \\ ${ }^{2}$ Department of Astronomy and Space Physics, Box 515, 75120 Uppsala, Sweden \\ ${ }^{3}$ Stockholm Observatory, AlbaNova University Center, 10691 Stockholm, Sweden
}

\begin{abstract}
Optical and near-IR surface photometry of the halos of disk galaxies and blue compact galaxies have revealed a very red spectral energy distribution, which cannot easily be reconciled with any normal type of stellar population. Using spectral evolutionary models, we demonstrate that a stellar population with an extremely bottom-heavy initial mass function can explain the red halos of both types of objects. Because of its very high mass-to-light ratio, this halo population may account for some of the missing baryons in the local Universe.
\end{abstract}

Keywords. galaxies: halos, galaxies: stellar content, dark matter

\section{The red halo mystery}

Deep optical/near-IR broadband images have revealed very faint halos around both edge-on disk galaxies (Zibetti, White \& Brinkmann 2004) and blue compact galaxies (Bergvall \& Östlin 2002; Bergvall, Marquart, Persson, et al. 2005). These halos, which are detected at surface brightness levels of $\mu_{g} \approx 28 \mathrm{mag} \operatorname{arcsec}^{-2}$ and $\mu_{B} \approx 26-27 \mathrm{mag}$ $\operatorname{arcsec}^{-2}$ respectively, have colours much too red to be reconciled with a normal, metalpoor stellar population like that in the halo of the Milky Way.

In Zackrisson, Bergvall, Östlin, et al. (2006), we use spectral evolutionary models to put various possible explanations for the red halo colours to the test. We find that the only scenario capable of explaining the red halos of both disk galaxies and blue compact galaxies is a stellar population obeying an extremely bottom-heavy $\operatorname{IMF}(\mathrm{d} N / \mathrm{d} M \propto$ $M^{-\alpha}$ with $\left.\alpha=4.50\right)$. Because of its very high mass-to-light ratio $\left(M / L_{B} \geqslant 40\right)$, this population effectively qualifies as baryonic dark matter and could account for some of the missing baryons in the local Universe.

To get to the heart of the red halo mystery, our group has recently started a large number of observational projects, including a search for red halos around post-starbursts (PI Zackrisson), a search for red halos around ellipticals (PI Bergvall), a continued search for red halos around blue compact galaxies (PI Östlin), a search for red halos around LSBGs in the SDSS (Caldwell \& Bergvall, these proceedings), integral-field spectroscopy of red halos (PI Bergvall), Spitzer observations of red halos (PI Bergvall) and direct tests of the bottom-heavy IMF hypothesis using star counts (PI Zackrisson).

\section{References}

Bergvall, N. \& Östlin, G. 2002, A\&A 390, 891.

Bergvall, N., Marquart, T., Persson, C., Zackrisson, E., \& Östlin, G. 2005, in: A. Renzini, \& R. Bender (eds.), Multiwavelength mapping of galaxy formation and evolution (Berlin: Springer), p. 355 .

Zackrisson, E., Bergvall, N., Östlin, G., Micheva, G., \& Leksell, M. 2006, ApJ 650, 812.

Zibetti, S., White, S. D. M. \& Brinkmann, J. 2004, MNRAS 347, 556. 